\title{
ADESÃO À DIETA MEDITERRÂNICA E CARACTERIZAÇÃO DO PERFIL ANTROPOMÉTRICO DE JOVENS ATLETAS
}

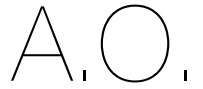

ARTIGO ORIGINAL

1 Escola Superior de Tecnologia da Saúde de Coimbra do Instituto Politécnico de Coimbra, Rua 5 de Outubro, $s / n$ 3046-854 Coimbra,

Portugal

‘Endereço para correspondência:

Teresa Ferreira

Escola Superior de Tecnologia da Saúde de Coimbra do nstituto Politécnico de Coimbra Rua 5 de Outubro, $s / n$ 3046-854 Coimbra, Portugal teresaclmferreira@gmail.com

Histórico do artigo:

Recebido a 1 de setembro de 2020 Aceite a 16 de abril de 2021

\begin{abstract}
ADHERENCE TO THE MEDITERRANEAN DIET AND CHARACTERIZATION OF THE ANTHROPOMETRIC PROFILE OF YOUNG ATHLETES
\end{abstract}

Teresa Ferreira'"; Helena Loureiro'; Margarida Pocinho

RESUMO

INTRODUÇÃo: A nutrição no futebol é crucial para um melhor rendimento desportivo e a Dieta Mediterrânica está comprovada como uma das mais adequadas para garantir o aporte de todos os nutrientes nas diferentes faixas etárias, promovendo o desenvolvimento das capacidades físicas do atleta.

OBJETIVOS: Caracterizar o perfil antropométrico e a prevalência do excesso de peso e obesidade, bem como avaliar a adesão à Dieta Mediterrânica de jovens atletas da formação do Vitória Sport Clube.

METODOLOGIA: No estudo participaram 33 jovens portugueses do sexo masculino, praticantes de futebol do Vitória Sport Clube na época 2019/2020, com idades compreendidas entre os 10 a 12 anos. Foi feita a medição do peso e altura, com consequente cálculo do Índice de Massa Corporal, e o perímetro da cintura para determinar o quociente entre este e a estatura para determinar o índice Waist-to-height ratio. Foi, ainda, aplicado por administração indireta, um questionário sobre características sociodemográficas, e um questionário validade e padronizado para avaliar a adesão à Dieta Mediterrânica, o índice KIDMED ${ }^{\circledR}$.

RESULTADOS: Da avaliação antropométrica, 63,6\% eram normoponderais, 33,3\% apresentavam excesso de peso e 3,0\% obesidade. Em relação ao perímetro da cintura, apenas 3,0\% da amostra encontrava-se acima do normal. Quanto ao questionário KIDMED ${ }^{\circledR}, 85,0 \%$ apresentava adesão alta à Dieta Mediterrânica e os restantes 15,0\% adesão moderada, ninguém apresentava adesão baixa.

CONCLUSõES: A prevalência do excesso de peso e obesidade foi de 36,3\% e a grande maioria apresentava adesão alta à Dieta Mediterrânica. O enfoque na educação alimentar deve ser considerado como estratégia nutricional major para maximizar a performance desportiva.

PALAVRAS-CHAVE

Dieta Mediterrânica, Estado nutricional, Futebol, Jovens atletas, Obesidade

\section{ABSTRACT}

INTRODUCTION: Soccer nutrition is crucial for a better sports performance and the Mediterreanean Diet is proven to be one of the most adequate to ensure the supply of all nutrients in different age groups, promoting the development of the athlete's physical capacities. OBJECTIVES: To characterize the anthropometric profile and the prevalence of overweight and obesity, as well as to evaluate the adherence to the Mediterranean Diet.

METHODOLOGY: The study included 33 young male Portuguese soccer players from Vitória Sport Clube in 2019/2020 season, aged between 10 and 12 years old. Weight and height were determined, through which the Body Mass Index was calculated, and the waist circumference to determine the quotient between it and height Waist-to-height ratio index. It was also applied by indirect administration, a questionnaire about sociodemographic characteristics and adherence to the Mediterranean Diet, KIDMED ${ }^{\circledR}$ index

RESULTS: From the anthropometric assessment, 63.6\% presented appropriate weight, 33.3\% were overweight and 3.0\% were obese. Regarding the waist circumference, only 3.0\% of the sample was above normal. As for the KIDMED ${ }^{\circledR}$ questionnaire, $85.0 \%$ had high adherence to the Mediterranean Diet and the remaining 15.0\% had moderate adherence, no one had low adherence. CONCLUSIONS: The prevalence of overweight and obesity was $36.3 \%$ and the vast majority had high adherence to the Mediterranean Diet. The focus on dietary education should be considered as a major nutritional strategy to maximize sports performance.

KEYWORDS

Mediterranean Diet, Nutrition status, Soccer, Young athletes, Obesity 


\section{INTRODUÇÃO}

A nutrição no desporto tem vindo a ganhar um grande destaque entre os clubes e federações como fator preponderante para melhorar e otimizar a performance em campo, permitindo alcançar as necessidades energéticas e nutricionais, manter uma boa composição corporal, melhorar e acelerar a recuperação e condição física, e minimizar o risco de lesão e doença (1-3). O futebol é um dos desportos mais conhecidos e com maior relevância em todo mundo. Requer um grande gasto energético, tanto nos treinos como nas competições, pois envolve diferentes intensidades de movimentos (curtos ou rápidos) e a utilização do metabolismo anaeróbico e aeróbico (4).

A Dieta Mediterrânica (DM) é apontada como uma das mais saudáveis, equilibrada, variada e capaz de suprir todas as necessidades de macronutrientes e micronutrientes necessários para um bom crescimento e desenvolvimento. Teve origem nos países que circundam o Mar Mediterrânico, sendo descrita, pela primeira vez, na década de 60 por Ansel Keys $(5,6)$. Caracteriza-se pelo grande consumo de produtos de origem vegetal, frescos, pouco processados e locais, tendo em conta a sazonalidade (7). Desde os anos 80 e até aos dias de hoje, a DM foi alvo de críticas positivas o que a levou a ser classificada como "Património Imaterial da Humanidade" pela United Nations Educational, Scientific and Cultural Organization (UNESCO) em 2010 (8). Para avaliar os hábitos alimentares de crianças e adolescentes tendo em conta este padrão, foi desenvolvido o Mediterranean Diet Quality Index for Children and Adolescents (índice KIDMED ${ }^{\circledR}$ ) (9).

O papel dos pais, família, treinadores e educadores é fundamental na aprendizagem do "saber comer", uma vez que, as crianças não estão dotadas a priori de conhecimentos para escolher os alimentos em função do seu benefício e valor nutricional (10). Assim, variáveis como o acesso a alimentos menos saudáveis, viver em zonas rurais ou urbanas, a instrução dos pais, a literacia alimentar e as suas práticas alimentares rotineiras vão influenciar diretamente o padrão alimentar das crianças. Lembrando que, estas aprendem através da observação dos adultos, vivenciando as suas escolhas, preparação e confeção dos alimentos $(10,11)$.

Assim sendo, uma avaliação da prevalência de excesso de peso e obesidade em clubes de formação e o estudo da associação entre as variáveis de interesse acima referidas nestas populações em específico, torna-se fundamental para a criação de novas abordagens e métodos de atuação e prevenção. Melhorar as práticas alimentares e qualidade de vida de crianças e adolescentes é um dos principais objetivos da saúde pública, tornando-os, não só melhores atletas através da otimização do seu desempenho e melhoria do rendimento desportivo, como, em última estância, futuros adultos mais saudáveis.

\section{OBJETIVOS}

Caracterizar o perfil antropométrico e a prevalência do excesso de peso e obesidade, bem como avaliar a adesão à Dieta Mediterrânica de jovens atletas da formação do Vitória Sport Clube (VSC).

\section{METODOLOGIA}

Foi realizado um estudo descritivo transversal, com a participação de 33 jovens atletas do sexo masculino, com idades compreendidas entre os 10 e os 12 anos, pertencentes aos escalões Benjamins B (10 anos), Benjamins A (11 anos) e Infantis (12 anos) da formação do VSC. A recolha de dados foi realizada no decorrer da época 2019/2020. Previamente à recolha de dados foi solicitada a autorização à Direção do VSC, bem como o consentimento informado para a participação neste estudo, aos encarregados de educação ou representantes legais de todos os atletas. O protocolo do estudo foi aprovado pela Comissão de Ética do Politécnico de Coimbra (CEPC). A cada atleta, aquando da avaliação, foram explicados os objetivos e procedimentos deste trabalho, bem como aos encarregados de educação no momento da resposta aos questionários. Os procedimentos utilizados respeitaram as normas internacionais da Declaração de Helsínquia (1975).

Os hábitos alimentares dos atletas foram avaliados através da adesão à DM, usando o índice KIDMED ${ }^{\circledR}$. Para isso, foi entregue um questionário validado e padronizado a cada participante, desenvolvido por SierraMajem et al (11). Este é constituído por 16 questões ("sim/não") de frequência alimentar. Cada questão tinha um score associado, respostas "sim" em questões com conotação negativa foram cotadas com -1 e com conotação positiva +1 . O score final é calculado através da soma dos valores atribuídos a cada questão, variando entre -4 e 12 . Este valor classificava a adesão à DM em três níveis: nivel 1 - alta adesão $(>8)$; nivel 2 - adesão intermédia (4-7); nível 3 - baixa adesão $(<3)$. Foi ainda aplicado um questionário para aferir alguns dados sociodemográficos, elaborado pela investigadora para determinar o grau de escolaridade completa do agregado familiar e a zona de residência.

A avaliação antropométrica foi realizada em vários dias, minutos antes de cada treino, sempre pela mesma pessoa, neste caso pela investigadora. Relativamente aos dados antropométricos, os atletas foram todos avaliados nas mesmas condições (apenas de calções e descalços), três vezes consecutivas para se obter um valor mais exato e um menor erro, segundo a Orientação n. ${ }^{\circ}$ 017/2013 da Direção-Geral da Saúde em Portugal (12). Para além disso, a avaliação antropométrica foi executada segundo os procedimentos padronizados pelo The Internacional Society for the Advancement of Kinanthropometry (ISAK) relativos à avaliação antropométrica, garantindo a uniformização das medições efetuadas (13). Para a pesagem foi utilizada a balança do modelo TANITA BC- $601^{\circledR}$. Era pedido a cada atleta que se colocasse em cima da balança com os pés afastados à largura dos ombros em posição antropométrica. Para a medição da estatura foi utilizado o estadiómetro do modelo Seca $217^{\circledR}$, era pedido ao atleta que se colocasse em posição antropométrica, com os pés juntos e a cabeça posicionada segundo o plano de Frankfurt. Para a medição do perímetro da cintura (PC) foi utilizada a fita métrica não extensível da marca CESCORF ${ }^{\circledR}$. A partir desta, foi possível obter-se o quociente entre o PC e a estatura (Waist-to-height ratio - WHtR). Esta classificação de percentis foi desenvolvida por Sardinha et al e permite avaliar o risco de desenvolvimento de doenças metabólicas relacionadas com a adiposidade abdominal. Tem um único ponto de corte para risco aumentado $(\geq 0,5)$, para crianças acima dos 5 anos e adolescentes (14). Para a classificação do estado nutricional foi utilizado como critério de referência as curvas de crescimento da Organização Mundial da Saúde (OMS) para o percentil e z-score (15). Primeiro foi calculado o Índice de Massa Corporal (IMC) através da fórmula de Quetelet: IMC = peso / (altura) 2 (16).

A análise estatística foi efetuada no programa Statistical Package for Social Sciences (SPSS) versão 23.0 para o Windows. Neste trabalho foi aplicada a Estatística Descritiva (Medidas de Tendência Central e de Dispersão, bem como Medidas de Frequências Absolutas e Relativas).

\section{RESULTADOS}

\section{Perfil Antropométrico}

Os dados antropométricos revelaram um peso médio de 35,26 kg (4,916), uma estatura média de 1,42 m (0,062), um IMC médio de 17,50 kg/m² $(1,737)$ e um PC médio de $62,58 \mathrm{~cm}(4,085)$. O valor médio do ratio wHtR, foi de $0,44(0,025)$ e apenas 1 participante se encontrava acima de 0,5 (Tabela 1). Verificou-se que em todas as faixas etárias, a maioria se apresentava entre os percentis 25 e 49, verificando-se um aumento deste com a idade (Tabela 2). De acordo com a interpretação dos pontos de corte dos z-scores e percentil do IMC para a idade, nenhum dos atletas 
apresentava magreza. Dos 63,6\% que se encontravam normoponderais, 11 pertenciam ao escalão Benjamim A, 7 ao escalão Benjamim B e 3 ao escalão Infantil. Quanto aos 33,3\% que apresentavam excesso de peso, 2 pertenciam ao escalão Benjamim A, 5 ao escalão Benjamim Be 4 ao escalão Infantil. Apenas 3,0\% da amostra apresentava obesidade, correspondendo a 1 atleta do escalão Infantil. Totalizando-se, assim, $36,3 \%$ da amostra com um $z$-score $\geq 1$ (percentil $\geq 85$ ). E 3,0\% da amostra com um $z$-score $\geq 2$ (percentil $\geq 97$ ). Verificou-se que o valor maior do IMC médio foi de $18,46 \mathrm{~kg} / \mathrm{m}^{2}(2,481)$ encontrado no escalão Infantil.

\section{Hábitos Alimentares e Caracterização da Adesão à Dieta} Mediterrânica

Foi possível destacar, positivamente, o consumo de uma peça de fruta

\section{Tabela 1}

Descrição dos dados antropométricos da amostra

\begin{tabular}{|c|c|c|c|c|}
\hline & MíNıмo & MÁXIMO & MÉDIA (்̇) & DESVIO-PADRÃO (S) \\
\hline Idade (anos) & 10,0 & 12,0 & 10,85 & 0,795 \\
\hline Peso (Kg) & 25,0 & 48,0 & 35,26 & 4,916 \\
\hline Estatura $(m)$ & 1,25 & 1,51 & 1,42 & 0,062 \\
\hline IMC $\left(\mathrm{kg} / \mathrm{m}^{2}\right)$ & 14,87 & 23,47 & 17,50 & 1,737 \\
\hline Perímetro da cintura $(\mathrm{cm})$ & 54,5 & 77,5 & 62,58 & 4,085 \\
\hline Ratio wHtR & 0,40 & 0,54 & 0,44 & 0,025 \\
\hline
\end{tabular}

IMC: Índice de Massa Corporal

wHtR: Waist-to-height ratio

\section{Tabela 2}

Dados relativos aos percentis do perímetro da cintura por idades

\begin{tabular}{|c|c|c|c|c|}
\hline \multirow{2}{*}{ PERCENTIL_PC } & \multicolumn{4}{|c|}{ IDADE (\%) } \\
\hline & 10 & 11 & 12 & TOTAL \\
\hline Abaixo P5 & 7,7 & 0 & 0 & 3,0 \\
\hline P6 a P9 & 0 & 0 & 0 & 0 \\
\hline P10 a P24 & 38,5 & 8,3 & 0 & 18,2 \\
\hline P25 a P49 & 53,8 & 75 & 50 & 60,6 \\
\hline P50 a P74 & 0 & 16,7 & 37,5 & 15,2 \\
\hline P75 a P84 & 0 & 0 & 0 & 0 \\
\hline P85 a P89 & 0 & 0 & 8,3 & 3,0 \\
\hline
\end{tabular}

P: Percentil

\section{Tabela 3}

Resposta dos Inquiridos ao questionário KIDMED ${ }^{\circledR}$

\begin{tabular}{|c|c|c|c|c|}
\hline & \multicolumn{2}{|c|}{ NÃO } & \multicolumn{2}{|c|}{ SIM } \\
\hline & $\mathbf{N}$ & $\%$ & n & $\%$ \\
\hline Consome uma fruta ou sumo de fruta todos os dias & 0 & $0 \%$ & 33 & $100,0 \%$ \\
\hline Consome uma segunda fruta todos os dias & 9 & $27,3 \%$ & 24 & $72,7 \%$ \\
\hline Consome produtos hortícolas frescos ou cozinhados regularmente, pelo menos uma vez por dia & 4 & $12,1 \%$ & 29 & $87,9 \%$ \\
\hline Consome produtos hortícolas frescos ou cozinhados regularmente, mais de uma vez por dia & 11 & $33,3 \%$ & 22 & $66,7 \%$ \\
\hline Consome peixe regularmente (pelo menos, 2 a 3 vezes por semana) & 6 & $18,2 \%$ & 27 & $81,8 \%$ \\
\hline Frequenta, mais de uma vez por semana, restaurantes de "fast-food" (hambúrguer) & 33 & $100,0 \%$ & 0 & $0 \%$ \\
\hline Consome leguminosas, mais de uma vez por semana & 4 & $12,1 \%$ & 29 & $87,9 \%$ \\
\hline Consome massa ou arroz, quase todos os dias (5 ou mais vezes por semana) & 8 & $24,2 \%$ & 25 & $75,8 \%$ \\
\hline Consome cereais ou produtos derivados de cereais (pão, etc.) ao pequeno-almoço & 5 & $15,2 \%$ & 28 & $84,8 \%$ \\
\hline Consome frutos oleaginosos (nozes, amêndoas, etc.) regularmente (pelo menos, 2 a 3 vezes por semana) & 23 & $69,7 \%$ & 10 & $30,3 \%$ \\
\hline Usa azeite em casa & 0 & $0 \%$ & 33 & $100,0 \%$ \\
\hline Costuma tomar o pequeno-almoço & 0 & $0 \%$ & 33 & $100,0 \%$ \\
\hline Consome laticínios (leite, iogurte, etc.) ao pequeno-almoço & 3 & $9,1 \%$ & 30 & $90,9 \%$ \\
\hline Consome produtos confecionados ou pastelaria ao pequeno-almoço & 31 & $93,9 \%$ & 2 & $6,1 \%$ \\
\hline Consome 2 iogurtes e/ou queijo $(40 \mathrm{~g})$ diariamente & 8 & $24,2 \%$ & 25 & $75,8 \%$ \\
\hline Consome doces ou guloseimas várias vezes por dia & 31 & $93,9 \%$ & 2 & $6,1 \%$ \\
\hline
\end{tabular}


Relação das respostas do questionário com o grau de escolaridade do agregado familiar

\begin{tabular}{|c|c|c|c|c|c|c|}
\hline & & & \multicolumn{4}{|c|}{ GRAU DE ESCOLARIDADE DO AGREGADO FAMILIAR } \\
\hline & & & ENSINO BÁSICO & $\begin{array}{c}\text { ENSINO } \\
\text { SECUNDÁRIO }\end{array}$ & ENSINO SUPERIOR & TOTAL \\
\hline \multirow{4}{*}{ Consome uma segunda fruta todos os dias } & \multirow{2}{*}{ Não } & $\mathrm{n}$ & 3 & 4 & 2 & 9 \\
\hline & & $\%$ & $50,0 \%$ & $23,5 \%$ & $20,0 \%$ & $27,3 \%$ \\
\hline & \multirow{2}{*}{ Sim } & $\mathrm{n}$ & 3 & 13 & 8 & 24 \\
\hline & & $\%$ & $50,0 \%$ & $76,5 \%$ & $80,0 \%$ & $72,7 \%$ \\
\hline \multirow{4}{*}{$\begin{array}{l}\text { Consome produtos hortícolas, pelo menos, uma vez } \\
\text { por dia }\end{array}$} & \multirow{2}{*}{ Não } & $\mathrm{n}$ & 2 & 2 & 0 & 4 \\
\hline & & $\%$ & $33,3 \%$ & $11,8 \%$ & $0,0 \%$ & $12,1 \%$ \\
\hline & \multirow{2}{*}{ Sim } & $\mathrm{n}$ & 4 & 15 & 10 & 29 \\
\hline & & $\%$ & $66,7 \%$ & $88,2 \%$ & $100,0 \%$ & $87,9 \%$ \\
\hline \multirow{5}{*}{ Consome produtos hortícolas mais de uma vez por dia } & \multirow{2}{*}{ Não } & $\mathrm{n}$ & 3 & 6 & 2 & 11 \\
\hline & & $\%$ & $50,0 \%$ & $35,3 \%$ & $20,0 \%$ & $33,3 \%$ \\
\hline & \multirow{3}{*}{$\operatorname{Sim}$} & $\mathrm{n}$ & 3 & 11 & 8 & 22 \\
\hline & & $\%$ & $50,0 \%$ & $64,7 \%$ & $80,0 \%$ & $66,7 \%$ \\
\hline & & $\%$ & $66,7 \%$ & $76,5 \%$ & $100,0 \%$ & $81,8 \%$ \\
\hline \multirow{4}{*}{ Consome leguminosas mais de uma vez por semana } & \multirow{2}{*}{ Não } & $\mathrm{n}$ & 1 & 4 & 0 & 4 \\
\hline & & $\%$ & $16,7 \%$ & $17,6 \%$ & $0,0 \%$ & $12,1 \%$ \\
\hline & \multirow{2}{*}{ Sim } & $\mathrm{n}$ & 5 & 14 & 10 & 29 \\
\hline & & $\%$ & $83,3 \%$ & $82,4 \%$ & $100,0 \%$ & $87,9 \%$ \\
\hline \multirow{4}{*}{ Consome cereais ou derivados ao pequeno-almoço } & \multirow{2}{*}{ Não } & $\mathrm{n}$ & 1 & 3 & 1 & 5 \\
\hline & & $\%$ & $16,7 \%$ & $17,6 \%$ & $10,0 \%$ & $15,2 \%$ \\
\hline & \multirow{2}{*}{ Sim } & $\mathrm{n}$ & 5 & 14 & 9 & 28 \\
\hline & & $\%$ & $83,3 \%$ & $82,4 \%$ & $90,0 \%$ & $84,8 \%$ \\
\hline \multirow{4}{*}{ Consome laticínios ao pequeno-almoço } & \multirow{2}{*}{ Não } & $\mathrm{n}$ & 0 & 2 & 1 & 3 \\
\hline & & $\%$ & $0,0 \%$ & $11,8 \%$ & $10,0 \%$ & $9,1 \%$ \\
\hline & \multirow{2}{*}{ Sim } & $\mathrm{n}$ & 6 & 15 & 9 & 30 \\
\hline & & $\%$ & $100,0 \%$ & $88,2 \%$ & $90,0 \%$ & $90,9 \%$ \\
\hline \multirow{4}{*}{ Consome 2 iogurtes e/ou queijo $(40 \mathrm{~g})$ diariamente } & \multirow{2}{*}{ Não } & $\mathrm{n}$ & 1 & 4 & 3 & 8 \\
\hline & & $\%$ & $16,7 \%$ & $23,5 \%$ & $30,0 \%$ & $24,2 \%$ \\
\hline & \multirow{2}{*}{ Sim } & $\mathrm{n}$ & 5 & 13 & 7 & 25 \\
\hline & & $\%$ & $83,3 \%$ & $76,5 \%$ & $70,0 \%$ & $75,8 \%$ \\
\hline \multirow{4}{*}{ Consome doces ou guloseimas várias vezes por dia } & \multirow{2}{*}{ Não } & $\mathrm{n}$ & 5 & 16 & 10 & 31 \\
\hline & & $\%$ & $83,3 \%$ & $94,1 \%$ & $100,0 \%$ & $93,9 \%$ \\
\hline & \multirow{2}{*}{ Sim } & $\mathrm{n}$ & 1 & 1 & 0 & 2 \\
\hline & & $\%$ & $16,7 \%$ & $5,9 \%$ & $0,0 \%$ & $6,1 \%$ \\
\hline
\end{tabular}

\section{Tabela 5}

Dados relativos ao grau de escolaridade e zona de residência do agregado familiar

\begin{tabular}{lcc}
\multicolumn{1}{c}{ GRAU DE ESCOLARIDADE } & FREQUÊNCIA (N) & $\begin{array}{c}\text { FREQUÊNCIA } \\
\text { RELATIVA (\%) }\end{array}$ \\
\hline Ensino Básico & 6 & $18,2 \%$ \\
\hline Ensino Secundário & 17 & $51,5 \%$ \\
\hline Ensino Superior & 10 & $30,3 \%$ \\
\hline Zona de residência & & \\
\hline Rural & 14 & $42,4 \%$ \\
\hline Urbana & 19 & $57,6 \%$ \\
\hline
\end{tabular}

hortícolas (ambas 75\%), leguminosas (75\%) e frutos oleaginosos (12,5\%), mas são os que mais consomem peixe (87,5\%), iogurtes e/ou queijo (87,5\%) (Tabela 3 e 4). Quanto à adesão à DM, não foi encontrado nenhum participante com baixa adesão, e a grande maioria $(84,8 \%)$ apresentavam adesão alta. O score de adesão médio foi de 9,42 $(1,838)$. O score de adesão mais elevado foi encontrado no escalão Benjamim B, 9,69 (1,932).

\section{Questões Sociodemográficas}

Em relação à constituição do agregado familiar, a maioria apresentava 4 pessoas na sua constituição [pai, mãe e irmão (a)]. Para o grau de escolaridade, foi tido em conta o mais elevado dentro do mesmo agregado familiar. Os participantes que tinham pais com o ensino superior apresentavam um IMC médio inferior, $16,98 \mathrm{~kg} / \mathrm{m}^{2}(1,569)$ e um score de adesão superior, 10,30 (1,567) (Tabela 5).

\section{DISCUSSÃO DOS RESULTADOS}

A maioria dos atletas avaliados é normoponderal, e 36,3\% apresenta excesso de peso e obesidade, o que está de acordo com outros estudos (17). Dados mais recentes do relatório Childhood Obesity Surveillance Initiative (COSI), relativos a Portugal, referem que o Norte apresenta cerca de $31,1 \%$ de excesso de peso e obesidade infantil, aproximando-se aos valores obtidos neste estudo, cujas crianças são todas residentes no Norte do país (18). Relativamente ao PC, verificou-se um aumento deste com a idade, dados estes corroborados no estudo desenvolvido por Sardinha et al (14).

Quanto à adesão à DM, de acordo com o índice $\mathrm{KIDMED}^{\circledR}, 84,8 \%$ da amostra revelou uma adesão alta, assemelhando-se a outros estudos realizados em faixas etárias semelhantes (19). Analisando o consumo de alimentos específicos típicos da DM, destacamos o consumo de pelo menos uma peça de fruta ser elevado, atingindo os 100\%, bem como o consumo de hortícolas, rondando os $88 \%$. Em ambos os casos, quando se questionou o segundo consumo de uma peça de fruta e 
hortícolas por dia, estas percentagens baixaram significativamente, mais de 20 pontos percentuais. No nosso estudo, cerca de $88 \%$ referiu consumir leguminosas mais do que 1 vez por semana, este resultado está bastante acima do encontrado nos estudos analisados $(7,20)$. Relativamente ao consumo de frutos oleaginosos a grande maioria da população em estudo não os consumia com regularidade, o que se encontra de acordo com os estudos analisados $(7,21)$. Seguindo a mesma tendência, quem obteve mais consumo foram os mais novos com pais com formação superior. Foi ainda possível constatar a existência de uma diferença tendencialmente significativa para uma diminuição da sua ingestão com o aumento da idade.

No que diz respeito ao consumo de iogurtes e/ou queijo, cerca de $75,8 \%$ referiu fazê-lo. Quanto ao consumo de doces ou guloseimas e à frequência de restaurantes de fast-food, todos responderam que não têm por hábito frequentá-los, foram encontrados valores semelhantes num estudo realizado numa academia de futebol (22). Por fim, alguns estudos encontraram uma associação positiva entre o grau de escolaridade superior dos pais com a adesão alta à DM, tanto nas crianças como nos adolescentes (23). O presente estudo parece seguir esta tendência, possivelmente por existirem mais conhecimentos sobre saúde, alimentação e estilos de vida, no entanto, são necessários mais estudos neste sentido. Por fim, outras referências apontam a zona de residência (rural ou urbana) como um determinante do nível de adesão à DM, em que a zona urbana apresentava uma maior adesão à DM $(7,22)$. A grande limitação deste trabalho reside no facto da amostra apresentar um tamanho reduzido, forçado pela baixa adesão na resposta aos questionários, possivelmente pela obrigatoriedade imposta destes serem enviado em formato digital. Assim, era expectável que o número de participantes fosse superior. No entanto, são levantadas algumas hipóteses para futuros estudos neste âmbito.

\section{CONCLUSÕES}

Os principais contributos destacados neste trabalho devem ser compreendidos adequadamente enquadrados nas limitações inerentes ao tamanho reduzido da amostra. Ainda assim, num panorama exploratório, é de destacar a importância do diagnóstico destas situações, impondo-se a continuidade do estudo dos padrões alimentares das crianças e jovens, permitindo em última instância a reflexão sobre os hábitos de vida da população portuguesa, essencial à promoção de boas práticas em todos os contextos de vida. É de salientar uma tendência para o regresso a uma alimentação marcada pela influência dos padrões mediterrânicos, surgindo algumas causas que podem estar na génese desta inversão: o aumento da literacia alimentar dos pais, consciencialização da importância da nutrição e alimentação para a saúde, introdução de alimentos do passado nos hábitos alimentares dos mais novos. Isto é fundamental para a diminuição da tendência nacional e mundial do crescimento de fenómenos como a obesidade infantil e diabetes tipo II. Destaca-se a importância major da inclusão de nutricionistas nas equipas multidisciplinares destes clubes, no sentido de intervirem nutricionalmente, monitorizarem o crescimento e a evolução nos escalões de formação, para assim termos jovens atletas mais saudáveis e podermos contribuir para uma melhor performance destes.

\section{REFERÊNCIAS BIBLIOGRÁFICAS}

1. Philippou E, Middleton N, Pistos C, Andreou E, Petrou M. The impact of nutrition education on nutrition knowledge and adherence to the Mediterranean Diet in adolescent competitive swimmers. Jornal of Science \& Medicine in Sport. 2017; 20(4):328-32.

2. IOC consensus statement on sports nutrition 2010. Journal of Sports Sciences,
2011; 29(S1): S3-S4.

3. Rodriguez NR, Di Marco NM, Langley S. American college of sports medicine position stand. Nutrition and athletic performance. Medicine \& Science Sports Exercise. 2009; 41:709-31.

4. Pinto, AP. Avaliação do estado de hidratação e rehidratação em atletas de futebol de ambos os sexos, de acordo com a ingestão de líquidos ad libitum, água simples e água com sal. Tese de Mestrado. Faculdade de Medicina da Universidade de Coimbra; 2014. 5. Santos, M. A qualidade da dieta mediterrânea numa população jovem do sul de Portugal. Porto (Portugal): Faculdade de Ciências da Nutrição e Alimentação da Universidade do Porto. 2002; Available from: https://repositorio-aberto.up.pt/ bitstream/10216/54706/5/67729_03-18T_TL_01_P.pdf.

6. Durão C, Oliveira J, de Almeida, MD. Portugal e o Padrão Alimentar Mediterrânico. Revista de Alimentação Humana. 2008; 14(3): 115- 128.

7. Mateus MP. Adesão ao Padrão Alimentar Mediterrânico em jovens no Algarve. Porto (Portugal): Faculdade de Ciências da Nutrição e Alimentação da Universidade do Porto. 2012; Available from: https://repositorio-aberto.up.pt/handle/10216/66678. 8. APN. Dieta Mediterrânica Um Padrão de Alimentação Saudável. [Internet]. Porto (Portugal). Associação Portuguesa de Nutrição. 2014; ISBN: 978-989-8631-152 Available from: https://www.apn.org.pt/documentos/ebooks/Ebook_Dieta_ Mediterranica.pdf.

9. Serra-Majem L, Ribas L, Ngo J, Ortega RM, Garcia A, Perez-Rodrigo C, et al. Food, youth and the Mediterranean diet in Spain. Development of KIDMED, Mediterranean Diet Quality Index in children and adolescents. Public health nutrition. 2004; 7(7):931-5. 10. Ansem WJ, Schrijvers CT, Rodenburg G, van de Mheen D. Is there na association between the home food environment, the local food shopping environment and children's fruit and vegetable intake? Results from the Dutch INPACT study. Public health nutrition. 2013; 16(7):1206 14; Available from: https://pubmed.ncbi.nlm.nih. gov/22874083/.

11. Rodrigues D, Muc M, Rodrigues PR, Pinto AM, Padez C. Dietary Patterns and Their Socioeconomic and Behavioral Determinants in 6- to 8-Year Old Portuguese Children. Ecology of food and nutrition. 2016; 1-14; Available from: http://handle. net/10316/31647.

12. Direcção-Geral da Saúde. Ministério da. Avaliação antropométrica no adulto Norma da DGS no 017/2013. Orientação [Internet]. 2013; 1-9. Available from: https:// www.dgs.pt/directrizes-dadgs/orientacoes-e circulares-informativas/orientacao n-0172013-de-05122013-pdf.aspx.

13. Marfell-Jones MJ OT, Stewart A, Lindsay Carter JE. International Standards for Anthropometric Assesmente, ISAK. 2006.

14. Sardinha L, Santos R, Vale S, Coelho e Silva M, Raimundo A, Moreira H, et al. Waist circumference percentiles for Portuguese children and adolescents aged 10 to 18 years. European Journal of Pediatrics. 2011; doi:10.1007/s00431-011-1595- 2. 15. Direcção-Geral Da Saúde RJ, Rito A, Breda J. Guia de Avaliação do Estado Nutricional Infantil e Juvenil [Internet]. 2011; Available from: www.insa.pt.

16. Quetelet LA. A treatise on man and the development of his faculties, 1842. Obesity Research. 1994; 2(1):72-85.

17. WHO. Portal do World Health Organization [Internet]. Adolescent obesity and related behaviours: trends and inequalities in the WHO European Region, 2002-2014. 2017; Available from: http://www.euro.who.int/_data/assets/pdf_file/0019/339211/ WHO_ObesityReport_2017_v3.pdf?ua=1.

18. COSI. Portal do Instituto Nacional de Saúde Doutor Ricardo Jorge e Serviço Nacional de Saúde [Internet]. Lisboa: Childhood Obesity Surveillance Initiative. 2019; Available from: http://www.insa.min saude.pt/cosi-portugal-2019-excesso-de-pesoe-obesidade-infantil continuam-em-tendencia-decrescente/.

19. Maria Lúcia M. Adesão ao Padrão Alimentar Mediterrânico de crianças a frequentar o $6^{\circ}$ ano de escolaridade de uma zona urbana da região do Porto. Porto (Portugal). 2016; Faculdade de Ciências da Nutrição e Alimentação da Universidade do Porto. 20. Almeida A. Adesão ao padrão alimentar mediterrânico e associação ao estado nutricional em crianças das escolas de $1^{\circ}$ ciclo da Póvoa de Lanhoso. Porto (Portugal). 2012; Faculdade de Ciências da Nutrição e Alimentação da Universidade do Porto Available from: https://repositorioaberto.up.pt/handle/10216/68552. 
21. Martins E, Mendes F, Fernandes R. Hábitos alimentares em crianças e jovens: nível de adesão à Dieta Mediterrânica. Viseu (Portugal). 2012; Escola Superior de Educação de Viseu. Available from: http://hdl.handle.net/10400.19/1074.

22. Azevedo BM. Antropometria, perceção corporal e adesão à dieta mediterrânica em crianças de uma escola de futebol. Porto (Portugal). 2017; Faculdade de Ciências da Nutrição e Alimentação da Universidade do Porto. Available from: https://repositorio aberto.up.pt/handle/10216/106791.

23. Rito A, Dinis A, Rascôa C, Maia A, Mendes S, Novais C, Lima J. Mediterranean Diet Index (KIDMED) Adherence, Socioeconomic Determinants, and Nutritional Status of Portuguese Children: The Eat Mediterranean Program. Portuguese Journal of Public Health. 2019; DOI:10.1159/000495803. 